\title{
FAMILIES OF SURFACES: HEIGHT FUNCTIONS AND PROJECTIONS TO PLANES
}

\author{
J. W. BRUCE, P. J. GIBLIN and F. TARI
}

\section{Introduction}

The work in this paper is part of our investigation of the geometry of surfaces in Euclidean 3 -space $R^{3}$, and the way this changes when the surfaces vary in 1-parameter families. In [5] we studied the geometry governed by contact with planes, by considering projection to lines. For a given smooth surface $M$ in $\mathrm{R}^{3}$ this is best described by the singularities of the height function on $M$. The height function fails to be stable (has an $A_{\geq 2}$ singularity) precisely at parabolic points. Cusps of Gauss are identified by the singularity of the height function being of type $A_{3}$. There is a natural two parameter family of height functions parametrised by the 2-sphere. The discriminant of this family is the dual of the surface, and the bifurcation set consists of the image of the parabolic set under the Gauss map. In [5] we use the theory of discriminants, bifurcation sets and functions on these sets to provide models of transitions on the parabolic set, its image in the Gauss sphere and of the dual when the surface varies in generic 1-parameter families. We also deal with the multi-local singularities.

Another aspect of the geometry, much studied over the last two decades, is that related to the contact with lines. This is captured by orthogonal projections of the surface to planes. The image of the set of points on $M$ where the direction of projection is tangent to the surface is the so-called profile (outline, apparent contour) of the surface. The profile can be described as the discriminant of a map-germ from the plane to the plane. A list of singularities of these mappings of codimension $\leq 6$ is given in 12] by Rieger, following earlier work of Arnold, Gaffney and Ruas, and du Plessis. In a subsequent paper, Rieger studied their related geometry [13].

So we have two families of projections, both parametrised by the 2-sphere. There is a striking duality result relating the bifurcation set of the family of projections to lines to that of the family of projections to planes, described in

Received January 30, 1996. 
[7, 2].We explain this duality in $\S 2$. We also shed some new light on Rieger's classification in section 2. For instance we relate this classification to that of singularities of functions on surfaces with boundary.

In $\S 3$ we determine modules of vector fields tangent to bifurcation sets of various germs from the plane to the plane, and then classify functions on such sets.

We complete in $\S 4$ the duality results given in $[7,2]$ to cover all the cases in Rieger's list and describe the correspondences between the transitions in 1-parameter families of the bifurcations sets of the height functions and those of orthogonal projections.

As a general reference for background results and terminology in singularity theory we recommend [14].

ACKNOWLEDGEMENTS. We acknowledge support from the Esprit grant VIVA, and the EPSRC grants GR/H 59855 and GR/J 28162.

\section{Projections of Surfaces.}

We first list the singularities of orthogonal projections which occur when considering 1-parameter families of surfaces. These have been given by Rieger in [12]; to be more precise he listed the singularities of mappings $\mathbf{R}^{2}, 0 \rightarrow \mathbf{R}^{2}, 0$ which have rank 1 and codimension $\leq 6$. The codimension here is that in the jet space.

We are interested in 1-parameter families of surfaces. So we need to consider a jet-extension map $X \times T \times S^{2} \rightarrow J^{k}\left(X, \mathrm{R}^{2}\right)$, where $X$ is the surface, moving with time $t \in T$ and $S^{2}$ parameterises the family of orthogonal projections. Consequently we are particularly interested in those mappings of codimension $\leq 5$ (there are 5 parameters in the source of this mapping). (As usual we need to consider not only germs of $\mathscr{A}$-codimension $\leq 5$ but also for example unimodular families of $\mathscr{A}$-codimension $\leq 6$.) It is not difficult to show that the mappings of codimension at most 5 in Rieger's list all arise when considering projections of 1-parameter families of surfaces in $R^{3}$.

THEOREM 2.1 (Rieger [12]). The following table lists all map-germs of corank 1 which will occur in a generic 3-parameter family of mappings from the plane to the plane. All but type 8 are simple, and $k>1$. We also list $C^{0}$-versal unfoldings, whose significance (and that of the last column) will be explained below. 


$\begin{array}{ccccc}\text { Type } & f(x, y) & \begin{array}{c}\text { codimension } \\ \text { of stratum }\end{array} & C^{0}-\text { versal unfolding } & g_{y} \\ 1 & (x, y) & 0 & & \\ 2 & \left(x, y^{2}\right) & 1 & & \text { sub } \\ 3 & \left(x, x y+y^{3}\right) & 2 & & \text { sub } \\ 4 k & \left(x, y^{3} \pm x^{k} y\right) & k+1 & \left(x, y^{3} \pm x^{k} y+\sum_{i=1}^{k-1} a_{i} x^{k-i-1} y\right) & A_{k-1} \\ 5 & \left(x, x y+y^{4}\right) & 3 & \left(x, x y+y^{4}+a y^{2}\right) & \text { sub } \\ 6 & \left(x, x y+y^{5} \pm y^{7}\right) & 4 & \left(x, x y+y^{5}+a y^{3}+b y^{2}\right) & \text { sub } \\ 7 & \left(x, x y+y^{5}\right) & 5 & \left(x, x y+y^{5}+a y^{3}+b y^{2}\right) & \text { sub } \\ 8 & \left(x, x y+y^{6} \pm y^{8}+\alpha y^{9}\right) & 5 & \left(x, x y+y^{6}+a y^{4}+b y^{3}+c y^{2}\right) & \text { sub } \\ 11_{2 k+1} & \left(x, x y^{2}+y^{4}+y^{2 k+1}\right) & k+2 & \left(x, x y^{2}+y^{4}+y^{2 k+1}+\sum_{i=1}^{k} a_{i} x^{k-i} y\right) & A_{1} \\ & \text { alternatively } & & & \\ & \left(x, x y^{2}+y^{4}+x^{k} y\right) & k+2 & \left(x, x y^{2}+y^{4}+x^{k} y+\sum_{i=1}^{k} a_{i} x^{k-i} y\right) & A_{1} \\ 12 & \left(x, x y^{2}+y^{5}+y^{6}\right) & 5 & \left(x, x y^{2}+y^{5}+a x y+b y^{3}+c y\right) & A_{1} \\ 16 & \left(x, x^{2} y+y^{4} \pm y^{5}\right) & 5 & \left(x, x^{2} y+y^{4}+a x y^{2}+b y^{2}+c y\right) & A_{2}\end{array}$

A key invariant associated to any family of mappings is its bifurcation set. This is the set of parameter values for which the associated mappings fail to be stable. In the dimension range (determined by Mather) where stable mappings are dense it will be diffeomorphic to a proper subanalytic subset of the unfolding space. For projections of a family of surfaces to planes we have a subset of $S^{2} \times \mathrm{R}$, consisting of pairs $(a, t)$ where the projection of the surface $X_{t}$ in the direction $a$ is not stable. Recall that the stable mappings from the plane to the plane were classified by Whitney, in [15], one of the founding papers of singularity theory. There are three types of stable mapping locally, namely the submersion, the fold, and the cusp. At a semi-local level the only type of interaction allowed is for two smooth parts of the image of the critical set to meet transversally. We can now describe the manner in which stability can fail, and consequently the various components of the bifurcation set.

In a family stability can fail when a map has (i) a rank 1 singularity $\mathscr{K}$ equivalent to $\left(x, y^{k}\right)$ where $k \geq 4$ (we shall refer to this as the swallowtail set, it corresponds geometrically to some line having 4-point or greater contact with $X_{t}$ ), (ii) a singularity of rank 0 (corank 2 set), (iii) a point of multiplicity three with the corresponding germ unstable, i.e. not a cusp (the lips/beaks set), (iv) a pair of tangent folds, i.e. when the images of two pieces of fold curve are tangent (the tangent folds set), (v) three concurrent fold points (triple-fold set), (vi) a fold and incident cusp (fold+cusp). Note that in each case one expects these sets to have codimension 1 in $S^{2} \times \mathrm{R}$, except in case (ii) which is of codimension 2. This stratum is in the closure of stratum (i), but it is of sufficient interest to be considered as a separate case. Since we are dealing with families of projections however there are no corank 2 singularities.

We now need to identify the corresponding bifurcation sets for the mappings given Theorem 2.1. Again there is a relevant paper of Rieger, namely 
[13]. The first three cases above are stable and there is no bifurcation set to consider. For the remainder we need to write out versal unfoldings, and locate the unstable points. This is a straightforward if messy business. Rieger also shows that in some of the cases one can consider much smaller unfoldings, construct their bifurcation sets and deduce that the bifurcation set of the full unfolding is homeomorphic to the product of the smaller unfolding with an affine space. (He uses the methods of [8], and also some of Damon's calculations.) We have a slightly different interest in the germs. In particular Rieger lumps together the swallowtail and lips/beaks strata, while it is important for us to separate them. Nevertheless his result (given in Theorem 2.1) is extremely useful.

So for example in case 6 above the bifurcation set of the original germ is homeomorphic to that of the given unfolding, and in case 7 the original bifurcation set is homeomorphic to the product of that of the given unfolding with a line. Similarly in case 8 (resp. 12 and 16) the term $y^{9}\left(\right.$ resp. $\left.y^{6}, y^{5}\right)$ in the second component of the germ is irrelevant for the unfolding.

We now wish to understand the changes in the viewgraph which occur in a 1-parameter family of surfaces. Our approach is the same as in [5]. Having identified the singularities which will arise (those of codimension at most 3) we need to find the associated bifurcation sets. These will sit inside the product of the view-sphere with the time axis, $S^{2} \times \mathrm{R}$. We then need to understand how this set projects down to the time axis, for this will give a description of the way in which the viewgraph changes with time. We are particularly interested in the subset of the bifurcation set corresponding to the lips/beaks and tangent fold strata, for reasons we now explain.

As we have said a crucial geometric invariant associated to any family of mappings is its bifurcation set. For functions, that is mappings whose target is the reals, stability fails via a degenerate singularity, or a pair of critical points sharing the same critical value. We shall refer to these subsets of the bifurcation set $\operatorname{Bif}(\mathrm{H})$ as the $A_{2}$ and $2 A_{1}$ strata respectively. If, as in [5], we consider the 2-parameter family of height functions on a surface, these correspond respectively to normals to the surface at parabolic points, and normals to bitangent planes.

In the paper [7] the first author and M. C. Romero-Fuster considered a curve or surface $X$ in Euclidean 3 -space $\mathrm{R}^{3}$ and the family of orthogonal projections of $X$ to lines and planes. The family of projections to lines is given by $H: X \times S^{2} \rightarrow \mathrm{R}, H(x, a)=\langle x, a\rangle$, where $\langle$,$\rangle is the usual inner pro-$ duct. For projections to planes let $B \subset S^{2} \times \mathrm{R}^{3}$ denote the set $\{(a, b):\langle a, b\rangle=0\}$ ( $B$ is the tangent bundle to $S^{2}$ ). Then the family is given by $P: X \times S^{2} \rightarrow B, P(x, a)=(a, x-\langle a, x\rangle a)$. As above we think of this as a family of maps $P_{a}: X \rightarrow \mathrm{R}^{2}$ parametrised by points of $S^{2}$. Projections to 
lines we will refer to as height functions, projections to planes simply as projections.

The main result in [7] showed that for a generic surface $X$ in $\mathrm{R}^{3}$ the bifurcation set of the family $H$ is dual to the lips/beaks and tangent fold subset of the bifurcation set of $P$. The duality here assigns to a smooth point $a$ of either bifurcation set the pole points $\pm a^{*}$ corresponding to the unique great circle (when viewed as an equator) tangent to the stratum at $a$. Note that the bifurcation sets are really subsets of the corresponding real projective planes, so we can identify these poles and lose the apparent ambiguity. In fact we work with the real projective planes, so that the tangent great circle is replaced by the tangent line.

So we consider the subset of the bifurcation set for the projections corresponding to lips/beaks and tangent folds. Our first result is a general one for corank 1 mappings of the plane.

Proposition 2.2. Let $f: \mathbf{R}^{2}, 0 \rightarrow \mathbf{R}^{2}, 0$ be a smooth germ of corank 1 with $\mathscr{A}$-versal unfolding given by $F: \mathrm{R}^{2} \times \mathrm{R}^{k}, 0 \rightarrow \mathrm{R}^{2}, 0$. Suppose without loss of generality that we write $f$ in the form $f(x, y)=(x, g(x, y))$, where $g$ is singular at the origin. Then that part of the bifurcation set of the germ $F$ corresponding to lips/beaks strata is diffeomorphic to the discriminant of the function germ given by $\partial g / \partial y: \mathbf{R}^{2}, 0 \rightarrow \mathrm{R}, 0$. (More precisely we choose a $\mathscr{K}$-versal unfolding of this function of dimension $k$ : the discriminant of this unfolding is diffeomorphic to the lips/beaks stratum of $F$.)

Proof. We can choose an unfolding of $f$ of the form $F(x, y, u)=$ $(x, G(x, y, u))$. Indeed by extending the number of variables we can ensure that $\partial G / \partial y$ is a $\mathscr{K}$-versal unfolding of $\partial g / \partial y$. For if $\partial g / \partial y+\sum u_{i} \phi_{i}(x, y)$ is a versal unfolding we can set $G=g+\sum u_{i} \int \phi_{i}(x, y) d y$. Now the lips/beaks stratum corresponds to the set of unfolding variables for which $\partial G / \partial y=0$ is singular, and this corresponds to the discriminant of $\partial G / \partial y$. We deduce that the lips/beaks subset of some versal unfolding of $f$ is diffeomorphic to the discriminant of $\partial g / \partial y$, so that in a miniversal unfolding the lips/beaks stratum is that of a $\mathscr{K}$-miniversal unfolding of $\partial g / \partial y$ crossed with an affine space of the relevant dimension.

The table of Theorem 2.1 contains, in the last column, the corresponding type of $\partial g / \partial y$, where $s u b$ is short for submersion. We can immediately deduce that the lips/beaks stratum is empty in cases $2,3,5,6,7,8$, is smooth of codimension 1 in cases $4_{2}, 11_{2 k+1}, 12$ and is a cuspidal edge in cases $4_{3}, 16$.

We now turn to the tangent folds stratum, in the cases 3, 5, 6, 7 and 8 . The point here is that we can arrange for the unfoldings to be of the form $\left(x, x y+y^{k}+\sum_{i=1}^{k-3} a_{i} y^{k-i-1}\right)=(x, g(x, y))$. Indeed it is a consequence of the 
complete transversal classification procedure in [4] that any finitely-determined germ $R^{2}, 0 \rightarrow R^{2}, 0$ which is not a fold, but which has smooth singular set, can be chosen to have 2-jet $(x, x y)$, and higher jet of the form $(x, x y+p(y))$ where $p(y)$ is a polynomial in $y$. Similarly its unfolding can be chosen to be of the form $(x, x y+P(y, u))$ where $P(y, 0)=p(y)$. The fact that we have tangent folds originating from points, say $\left(x, y_{1}\right)$ and $\left(x, y_{2}\right)$, means that the partial derivatives of the second component with respect to $x$ at the two points are equal. But this would then show that $y_{1}=y_{2}$, a contradiction. In other words all germs with 2-jet $(x, x y)$ have tangent folds set which is empty. We deduce

Proposition 2.3. The germs of type $2,3,5,6,7,8$ have empty lips/beaks and tangent folds strata.

In other words these germs are invisible to the height function geometry. The computations of the bifurcation sets for the remaining cases are dealt with in [13]. We reformulate some of the results there in terms of singularities of functions on surfaces with boundary.

THEOREM 2.4 (Compare [13]).

(i) For the germs $4_{k}$ the beaks/lips subset of the bifurcation set is diffeomorphic to the discriminant of an $A_{k-1}$ singularity.

(ii) For the germs $11_{2 k+1}$ the beaks/lips/tangent folds subset of the bifurcation set is diffeomorphic to (a subset of) the discriminant of a function on a manifold with boundary, namely that of type $C_{k}$ with normal form $g(x, y)=x y+x^{k}$, where the boundary is given by $y=0$.

(iii) For the germ of type 12 the beaks/lips/tangent folds subset of the bifurcation set is diffeomorphic to the discriminant of a function on a manifold with boundary, namely that of type $B_{2}$, with normal form $x^{2}+y^{2}$, where the boundary is given by $y=0$ again.

(iv) For the germ of type 16 the beaks/lips tangent folds subset of the bifurcation set is diffeomorphic to the discriminant of $x^{2}+4 y^{3}+2 a x y+2 b y+c$ as a function on the manifold with boundary given by $y=0$.

Proof. For the first result note that these germs have local multiplicity 3 (they are $\mathscr{K}$-equivalent to the germs $\left(x, y^{3}\right)$ ) so cannot deform to tangent fold singularities. The beaks lips stratum is easily obtained. The second part is also a straight calculation using the alternative form of the germ and its unfolding. We give the details of part (iii), that is we consider the germ $\left(x, x y^{2}+y^{5}+a x y+b y^{3}+c y\right)$. The lips/beaks stratum is the discriminant of $2 x y+5 y^{4}+a x+3 b y^{2}+c$, which can be parametrised as the set 


$$
\mathrm{BL}=\left\{\left(a, b,-\frac{5}{16} a^{4}-\frac{3}{4} a^{2} b\right)\right\}
$$

while the tangent folds stratum is given by

$$
\mathrm{TF}=\left\{\left(a,-2 y^{2}-2 a y-3 a^{2}, y^{4}+2 a y^{3}+3 a^{2} y^{2}+2 a^{4}\right)\right\} .
$$

TF is a surface with boundary, the boundary, being given by the image of $b+(1 / 2) a=0$, is parametrised as $\left\{\left(a,-(5 / 2) a^{2},(25 / 16) a^{4}\right)\right\}$. This curve also lies in the BL stratum, as one can easily check. The two surfaces TF and BL are tangential along this common boundary and it is not difficult to show that their union is diffeomorphic to the union of the sets given in $(a, b, c)$ space by $\{b=0\}$ and $\left\{b=a^{2}: a \geq 0\right\}$. This is the discriminant of another function on a manifold with boundary, namely one of type $B_{2}$.

Clearly the discriminant is a local product. We now determine the nature of the singularities corresponding to intersection of the two strata. There is a natural $\mathrm{R}^{*}$-action on the unfolding space: for a given $\lambda \neq 0$ we change coordinates in the source by $(x, y) \mapsto\left(\lambda^{-3} x, \lambda^{-2} y\right)$ and in the target by $(u, v) \mapsto\left(u, \lambda^{5} y\right)$; this gives an action on the unfolding space via $(a, b, c) \mapsto\left(\lambda a, \lambda^{2} b, \lambda^{4} c\right)$. So the intersection is the union of $(0,0,0)$ and a single orbit through, say, $(2,10,25)$. Calculation shows that $\left(x, x y^{2}+y^{5}+2 x y-10 y^{3}+25 y\right)$ has an $11_{5}$ singularity at $(x, y)=(20,-1)$. So the intersection consists of such singularities.

\section{The multilocal singularities.}

The multilocal singularities of mappings $R^{2} \rightarrow R^{2}$ have also been studied. The classifications so far however have concentrated on multigerms of $\mathscr{A}$ codimension $\leq 4$; see for example [9]. The duality correspondence for these germs is given in [2,7]. For our investigation we also need multigerms of $\mathscr{A}$ codimension 5 that correspond to codimension 3 multi-local singularities of the height function. We shall not classify all these singularities but investigate only those relevant to the duality results. These are (i) 4-point contact folds, (ii) triple tangent folds, (iii) tangent lips/beaks plus fold.

The multigerm (i) is part of the series of $k$-point contact folds represented by $\left\{\left(x_{1}, y_{1}^{2}\right) ;\left(x_{2}, y_{2}^{2} \pm x_{2}^{k}\right)\right\}$. Its bifurcation set (which consists only of tangent fold points) is the discriminant of an $A_{k}$ singularity [2].

The case (ii) can be represented by $\left\{\left(x_{1}, \pm y_{1}^{2}\right) ; \quad\left(x_{2}, y_{2}^{2} \pm x_{2}^{2}\right)\right.$; $\left.\left(x_{3}, y_{3}^{2}+a x_{3}^{2}+b x_{3}^{3}\right)\right\}$ where $a, b$ are moduli $(b \neq 0)$. A versal unfolding has the form

$$
\left\{\left(x_{1}, y_{1}^{2}\right) ;\left(x_{2}, y_{2}^{2}+x_{2}^{2}+c\right) ;\left(x_{3}+c, y_{3}^{2}+a x_{3}^{2}+b x_{3}^{3}+e\right)\right\} .
$$

For fixed values of $a, b$ the bifurcation set consists of three transverse planes. 
The case (iii) is given by $\left\{\left(x_{1}, y_{1}^{2}\right) ;\left(x_{2}, x_{2}^{2}+y_{2}^{3} \pm x_{2}^{2} y_{2}\right)\right\}$, with a versal unfolding of the form $\left\{\left(x_{1}, y_{1}^{2}\right) ;\left(x_{2}, x_{2}^{2}+y_{2}^{3} \pm x_{2}^{2} y_{2}+a x_{2}+b y_{2}+c\right)\right\}$. The lips/ beaks stratum consists of the plane $\{(a, 0, c)\}$ while the tangent folds stratum is given by

$$
\left\{\left(-2 x_{2} \mp 2 x_{2} y_{2},-3 y_{2}^{2} \mp x_{2}^{2}, x_{2}^{2}+2 y_{2}^{3} \pm 2 x_{2}^{2} y_{2}\right)\right\} \text {. }
$$

This is a cuspidal edge intersecting the lips/beaks stratum in a node or an isolated point. The full bifurcation set is diffeomorphic to the variety $\left(z \pm y^{2}\right)\left(z^{3}-x^{2}\right)=0$.

\section{Sections of bifurcation sets.}

We now need to determine the generic sections of these bifurcation sets, which arise when we project them from $S^{2} \times \mathrm{R}$ to R. For those singularities whose bifurcation sets are discriminants this has already been done (see [1]). In particular we need not concern ourselves with the germs of type $4_{k}$. Similarly one can classify projections of discriminants of functions on a manifold with boundary [10]. As an illustration we shall deal with the cases 16 in Theorem 2.1 whose bifurcation set is described in Theorem 2.4, and the "tangent lips/beaks plus fold" in the multilocal situation. We shall need the following result.

Let $f=\left(f_{1}, \ldots, f_{n}\right): \mathbf{C}^{n}, 0 \rightarrow \mathbf{C}^{n}, 0$ be a holomorphic map which is finite, that is the inverse image of 0 is locally 0 . Let $\mathcal{O}_{n}$ be the set of holomorphic function germs $\mathrm{C}^{n}, 0 \rightarrow \mathrm{C}$ and let $F:\left(\mathcal{O}_{n}\right)^{n} \rightarrow \mathcal{O}_{n}$ be the homomorphism defined by $F\left(g_{1}, \ldots, g_{n}\right)=\sum_{i=1}^{n} g_{i} f_{i}$. Then the kernel of this homomorphism is generated by the obvious vectors, namely $f_{i} e_{j}-f_{j} e_{i}$, where as usual the vector $e_{i}$ has a 1 in the $i^{\text {th }}$ place and 0's elsewhere. This follows easily from the fact that $f_{1}, \ldots, f_{n}$ form an $\mathcal{O}_{n}$-regular sequence.

4.1. Generic sections of $V_{1}=\left\{(x, y, z):\left(z^{2}-y^{3}\right)\left(8 z+x^{2} y-\frac{1}{432} x^{6}\right)=0\right\}$.

We seek the vector fields tangent to the variety $V_{1}$ which is the bifurcation set of case 16 (Theorem 2.4 (iii)). The way to do this is to consider the two components of this variety. The subset corresponding to the lips/beaks is given by $z^{2}=y^{3}$. The set of tangent vector fields to this variety is generated by

$$
e_{11}=\partial / \partial x, e_{12}=2 y \partial / \partial y+3 z \partial / \partial z, e_{13}=2 z \partial / \partial y+3 y^{2} \partial / \partial z \text {. }
$$

On the other hand those tangent to the variety given by the vanishing of the second equation are generated by

$$
\begin{aligned}
& \left.e_{21}=x \partial / \partial x+4 y \partial / \partial y+6 z \partial / \partial z, e_{22}=-8 \partial / \partial x+\left(2 x y-\frac{1}{72}\right) x^{5}\right) \partial / \partial z, e_{23}= \\
& -8 \partial / \partial y+x^{2} \partial / \partial z
\end{aligned}
$$


We seek the intersection of the modules generated by these vector fields. A short calculation shows that we are seeking the kernel of the homomorphism $\mathcal{O}_{3}^{3} \rightarrow \mathcal{O}_{3}$ given by

$$
\begin{aligned}
\left(g_{1}, g_{2}, g_{3}\right) \mapsto g_{1} f_{1}+g_{2} f_{2}+g_{3} f_{3} & =g_{1}\left(3 z+\frac{1}{4} y x^{2}\right)+g_{2}\left(2 x y-\frac{1}{72} x^{5}\right) \\
& +g_{3}\left(-3 y^{2}-\frac{1}{4} z x^{2}\right) .
\end{aligned}
$$

But the map $\left(g_{1}, g_{2}, g_{3}\right): \mathrm{C}^{3}, 0 \rightarrow \mathrm{C}^{3}, 0$ is clearly finite, and the result above will apply. Substituting back we find that the required vector fields are

$$
\begin{aligned}
& \psi_{1}=x \partial / \partial x+4 y \partial / \partial y+6 z \partial / \partial z \\
& \psi_{2}=-8 f_{1} \partial / \partial x+f_{2}(2 y \partial / \partial y+3 z \partial / \partial z) \\
& \psi_{3}=f_{3}(2 y \partial / \partial y+3 z \partial / \partial z)+f_{1}\left(2 z \partial / \partial y+3 y^{2} \partial / \partial z\right) \\
& \psi_{4}=8 f_{3} \partial / \partial x+f_{2}\left(2 z \partial / \partial y+3 y^{2} \partial / \partial z\right) .
\end{aligned}
$$

These (real) vector fields will be tangent to the real part of the bifurcation set. Changes of coordinates preserving this set are obtained by integrating linear combinations of the $\psi_{i}$.

We can now classify generic functions on this bifurcation set, using the usual techniques, in particular the idea of a complete transversal [4]. Here we need to consider non-stable functions. Let $\mathscr{G}$ be the group of pairs of diffeomorphisms $(h, k)$ where $h$ preserves the variety $V_{1}$ in the source and $k$ is any change of coordinates in the target. We obtain the following result.

THEOREM 4.1. On the variety $V_{1}$ given above there is a unique stable function up to $\mathscr{G}$-equivalence. This has normal form $x+y$. The next least degenerate germ has normal form $x$, and this function is topologically equivalent to the stable germ $x+y$. The next least degenerate germ after this has two moduli and is of the form $y \pm z+a x^{2}+b x^{3}, a \neq 0$. The sections of the variety by this germ are illustrated in Figure 1 for $a>0$ and $a<0$.

Proof. The tangent space to the $\mathscr{G}$-orbit of a germ of a function $f$ on $V_{1}$ is

$$
T \mathscr{G} f=\mathscr{E}_{3}<\psi_{1}(f), \psi_{2}(f), \psi_{3}(f), \psi_{4}(f)>+f^{*}\left(\mathscr{M}_{1}\right)
$$

where $\psi_{i}(i=1, . ., 4)$ are as above, $\mathscr{E}_{n}$ the ring of germs from $\mathbf{R}^{n}, 0 \rightarrow \mathbf{R}, \mathscr{M}_{n}$ the maximal ideal in $\mathscr{E}_{n}$ and $f^{*}\left(\mathscr{M}_{1}\right)$ the pull back of $\mathscr{M}_{1}$ by $f$. The classification is carried out inductively in the jet space $J^{k}(3,1)$ for low values of $k$ using the complete transversal method and determinacy results described in [4] together with Mather's Lemma. Details are left to the reader.

We need criteria for recognising the types of sections we get on the bifurcation set of a family of projections when the singularity at the origin is of type 16 . 
Lemma 4.2. Let $W$ be a variety diffeomorphic to $V_{1}$. Then a function $f: W \rightarrow \mathrm{R}$ yields sections equivalent to those given by $y \pm z+a x^{2}+b x^{3}$ on $V_{1}$ if and only if

(i) $f$ is a submersion whose fibre at the origin is transverse to the smooth stratum of $W$, and

(ii) the sections of $f$ on the cuspidal edge in $W$ are of Morse type.

This follows by an analysis of the conditions in the proof of the previous theorem for a function to be $\mathscr{G}$-equivalent to $y \pm z+a x^{2}+b x^{3}$.

4.2. Generic sections of $V_{2}=\left\{(x, y, z):\left(z \pm y^{2}\right)\left(z^{3}-x^{2}\right)=0\right\}$.

The variety $V_{2}$ represents the bifurcation set of the "lips/beaks plus tangent fold" multi-local singularity. We need to find the vector fields tangent to this variety. We proceed as in the previous case and start by finding the generators for the vector fields tangent to the varieties $z \pm y^{2}=0$ and $z^{3}-x^{2}=0$.

For $z^{3}-x^{2}=0$ these are given by $e_{11}=\partial / \partial y, \quad e_{12}=3 x \partial / \partial x+$ $2 z \partial / \partial z, e_{13}=3 z^{2} \partial / \partial x+2 x \partial / \partial z$, while for variety given by $z \pm y^{2}=0$ they are generated by $e_{21}=\partial / \partial x, e_{22}=y \partial / \partial y+2 z \partial / \partial z, e_{23}=\partial / \partial y \mp 2 y \partial / \partial z$.

The intersection of the corresponding modules (those fields tangent to the variety $V_{2}$ ) is generated by

$$
\begin{aligned}
& \psi_{1}=3 x \partial / \partial x+y \partial / \partial y+2 z \partial / \partial z \\
& \psi_{2}=\left(x^{2}-z^{3}\right) \partial / \partial x \\
& \psi_{3}= \pm 3 x y \partial / \partial x-z \partial / \partial y \pm 2 y z \partial / \partial z \\
& \psi_{4}=\mp 3 y z^{2} \partial / \partial x+x \partial / \partial y \mp 2 x y \partial / \partial z .
\end{aligned}
$$

We can now classify generic functions on the variety $V_{2}$ as we did in Theorem 4.1.

THEOREM 4.3. On the variety $V_{2}$ there is a unique stable function up to changes of coordinates in the source which preserves the variety and changes of coordinates in the target. This has normal form $y$. The next least degenerate germ has one modulus and is of the form $x+z+a y^{2}$ where $a \neq 0, \pm 1$. The sections of the variety by this germ are illustrated in Figure 2.

Here we need to recognise the type of sections of the bifurcation set of a family of projections when the multi-singularity at the originis of type "tangent lips/beaks plus fold". Here the projection to the time coordinates is also not generic (even for a generic family of surfaces). Recall that on its bifurcation set the tangent beaks (res. lips) plus fold is a distinguished point, corresponding to a point of tangency between a cuspidal edge and a smooth surface. We claim that the section through the distinguished point of the time $t=$ constant sphere is also tangent to the cuspidal edge. In the case of 
the tangent lips plus fold this is clear. The lips grow into a lips proper in one time direction only, and only then give rise to any tangent folds. If the section were not tangent to the cuspidal edge there would be a cusp of tangent fold points before, during and after the lips transitions. Similarly for the tangent beaks plus fold. It is clear using the unfolding given in 2.1 that the tangent folds stratum has cusps (corresponding to triple tangent folds) at nflexions of the perturbed beaks, and these only occur in the direction in which the beaks split into two cusps. We shall prove these results in the next section.

Lemma 4.4. Let $W$ be a variety diffeomorphic to $V_{2}$. Then a function $f: W \rightarrow \mathrm{R}$ yields sections equivalent to those given by $x+z+a x^{2}$ on $V_{2}$ if and only if

(i) $f$ is a submersion whose fibre at the origin is transverse to the smooth stratum of $W$.

(ii) The sections of $f$ on the cuspidal edge in $W$ are of Morse type.

This follows from the criterion in the proof of Theorem 4.3 for a function to be $\mathscr{G}$-equivalent to $x+z+a x^{2}$.

\section{Duality and height functions.}

In this section we match up the singularities of the projections to those of the height functions. This was done for a number of cases in [7] and [2], but a wide variety of interesting cases remain.

We recall that for the case of monogerms the unstable singularities of height functions that occur generically in 1-parameter families of surfaces are $A_{2}, A_{3}, A_{3}^{ \pm}, A_{4}, D_{4}^{ \pm}$. For the multilocal case they are $A_{1}^{3}, A_{1} A_{2}, A_{1}^{4}, A_{1}^{2} A_{2}$, $A_{1} A_{3}, A_{2}^{2}$, see [5]. The local singularities for the projections are as in Theorem 2.1. The multilocal ones that are of interest here are those enumerated in [7] together with those given in $\S 3$.

It is shown in [7] that the dual of an $A_{2}$ point is a lips/beaks $\left(4_{2}\right.$ in Theorem 2.1). More generally, a result in [2] (Theorem 2.1 (i)) states that the tangent line has $k$-point contact with the $A_{2}$ stratum if and only if the dual direction determines a projection of type $4_{k}$. In [7] the dual of an $A_{3}$ is a gulls $\left(11_{5}\right)$. For the multilocal correspondence, the dual of a $2 A_{1}$ is a tangent folds singularity [7]. Here the tangent line has $k$-point contact with the $2 A_{1}$ stratum if and only if the dual direction determines a projection of type k-point contact fold [2] (Theorem 2.1 (ii)).

We shall describe in this section the duality correspondence for the remaining cases in Theorem 2.1 and those of the local and multi-local singularities of the height functions that occur in generic 1-parameter families of 